\title{
Magnetic Oscillations and Maxwell Theory
}

\author{
Artur Sowa \\ Department of Mathematics \\ Graduate Center of The City University of New York \\ 33 West 42nd Street \\ New York, NY 10036 \\ sow@clsc2.gc.cuny.edu
}

\begin{abstract}
We explore the possibility of using the Kaluza-Klein geometry of Riemannian Submersions to modify the classical Maxwell Theory. We further argue that the resulting modification of Electromagnetismcf. equations (3) as well as (4) and (5) - may be interesting in the context of, among other topics, magnetic oscillations in metals.
\end{abstract}

1. The Maxwell equations are geometric and can be expressed as equations defining a principal $U(1)$ connection. Such a connection $A$ is a differential 1-form on the total space of a $U(1)$-bundle, which in addition satisfies

$$
R_{\theta}^{*} A=0, \quad A\left(\frac{\partial}{\partial \theta}\right)=1 .
$$

Here $\theta \in U(1), R_{\theta}$ denotes the $U(1)$-action on the bundle and $\frac{\partial}{\partial \theta}$ is the canonical vertical vector field induced by this action. Now if $F_{A}=d A$ is the curvature, then (in the absence of nonstatic charge distributions) the Maxwell equations become

$$
d F_{A}=0, \quad d^{*}\left(f F_{A}\right)=0,
$$

where $f$ is the so called material constant (like the dielectric constant, the inverse of the magnetic permeability, etc.) 1

\footnotetext{
${ }^{1}$ In what follows all physical constants irrelevant for our discussion are set to 1.
} 
2. It has been established by the Aharonov-Bohm experiment that the connection itself has a physical meaning. Thus we see that the equations (11) are themselves a part of Maxwell Theory. Clearly, the first of the equations states $U(1)$-symmetry. What is the significance, if any, of $A\left(\frac{\partial}{\partial \theta}\right)=1$, though? - We will try to suggest an answer to this question.

Let us recall that principal connections also have another interpretation. A principal bundle naturally distinguishes the vertical direction $\frac{\partial}{\partial \theta}$, but distinguishing a complimentary distribution of horizontal planes requires an additional bit of structure - a connection. Such a horizontal distribution is usually described as $\operatorname{ker} A$.

If we replace $A$ by $\tilde{A}=f \cdot A$, where $f$ is a positive function constant along the fibers, then $\operatorname{ker} \tilde{A}=\operatorname{ker} A$, and $\tilde{A}$ defines the same horizontal distribution as $A$. We will call $\tilde{A}$ a connection with amplitude.

It has to be decided, which is the physical notion of a connection? Is it the "pure" horizontal plane distribution (and the Aharonov-Bohm experiments do not go beyond it), or is it the 1 -form $A$ or maybe $\tilde{A}-$ i.e. there are a priori more than just one choice of $A$ ?

We argue in this article that the choice of $\tilde{A}$ is a very interesting one. Moreover, we propose to interpret $f=\tilde{A}\left(\frac{\partial}{\partial \theta}\right)$ as a material "constant", which in addition will be allowed to vary according to a certain rule.

3. In the meantime, we want to ask if there is a natural candidate for a system of geometric equations for $f A$, such that the Maxwell equations are a "limiting" case.

Kaluza and Klein were the first to observe that the structure induced by a connection on a principal fiber bundle allows us to lift the metric tensor of space-time to a metric tensor on the total space of the bundle.

The tangent space of the total space of a principal bundle $\pi: P \rightarrow M$ at each point decomposes into the vertical, i.e. tangent to the fiber, and the horizontal part defined by the connection. We declare them orthogonal. The scalar product $\mu=\mu(A)$ is defined by

$$
\mu(X, Y)=A(X) A(Y)+g\left(\pi_{\star} X, \pi_{\star} Y\right),
$$

where $g$ is the metric tensor on $M$. 
This metric defines the Laplace-Beltrami operator on forms

$$
\triangle_{A}=d \delta+\delta d,
$$

where $d$ is the exterior derivative and its formal adjoint $\delta$ acting on forms of degree $q$ is defined by $\delta=(-1)^{\operatorname{dim} P(q+1)+1} \star d \star$ (where $\star$ denotes the $\mu$-Hodge star and therefore depends on $A$ ).

We postulate that the elliptic eigenvalue problem

$$
\triangle_{A}(f A)=\nu f A
$$

is a natural choice for a description of static states of the connection with amplitude. This equation was introduced in [3] and further studied in [四] (with a wrong numerical constant) and [5].

4. Our aim now is to explicitly evaluate this Laplacian on the form $f A$. We assume for simplicity that $M$ is orientable, but this assumption is not necessary. Also $\operatorname{dim} M=m$.

Let us first notice that $d V_{P}=d V_{M} \wedge A$, where $d V_{P}$ is the volume element of $P$ and $d V_{M}$ is the pull-back of the volume element of $M$ to $P$. (In what follows we will often identify differential forms on $M$ with their pull-backs to $P$.) Thus, in particular, $d V_{P}$ does not depend on the choice of a connection $A$. Indeed, if $A^{\prime}$ is another connection, then $A^{\prime}=A+d a$ and $d V_{M} \wedge A^{\prime}=d V_{M} \wedge A+d V_{M} \wedge a=d V_{M} \wedge A$. Now, if $\alpha$ is a horizontal $p$-form on $P$, i.e. $\alpha\left(\frac{\partial}{\partial \theta}\right)=0$, then we have

$$
\begin{gathered}
\star \alpha=\star_{M} \alpha \wedge A, \\
\star(\alpha \wedge A)=(-1)^{m-p} \star_{M} \alpha,
\end{gathered}
$$

where $\star_{M}$ is the Hodge star of $g$ (on $M$ ). In particular $\star(f A)=$ $(-1)^{m} f d V_{M}$ and, since $f$ is constant along fibers, we see that the 1 -form $f A$ is co-closed. We have

$$
\begin{gathered}
d(f A)=d f \wedge A+f F_{A}, \\
\star d(f A)=(-1)^{m-1} \star_{M} d f+f \star_{M} F_{A} \wedge A, \\
d \star d(f A)=(-1)^{m-1} d \star_{M} d f+d\left(f \star_{M} F_{A}\right) \wedge A+(-1)^{m-2} f \star_{M} F_{A} \wedge F_{A},
\end{gathered}
$$


$\delta d(f A)=(-1)^{m-1} \star d \star d(f A)=\left(-\triangle f+|F|^{2} f\right) \wedge A+(-1)^{m} \delta_{M}\left(f F_{A}\right)$.

Thus the equation (3) is equivalent to the following system of equations (on $\mathrm{M}$ )

$$
\begin{gathered}
\delta_{M}\left(f F_{A}\right)=0 \\
-\triangle f+\left|F_{A}\right|^{2} f=\nu f .
\end{gathered}
$$

(Note that in this paper $\triangle \leq 0$ on functions, and $\triangle \geq 0$ on 1-forms.)

It should be noted that these equations are not the Euler-Lagrange equations corresponding to a functional in the two variables $f$ and $A$.

5. It is clear that $f$ plays the role of a "material constant". It has long been known from experiments that at low temperatures and high magnetic fields, material constants lose their meaning as simple coefficients of proportionality. It was determined that in such conditions the relation between, say, magnetic field $H$ and magnetic induction $H=\frac{1}{\mu} B$ does no longer hold and should be replaced by a more general relation $H=H(\mu, B)$. (This one too, though, has a very limited range of applications.) Moreover, in such extreme conditions new phenomena have been observed, which could not be explained by the Maxwell Theory and were attributed quantum character. Here we have in mind all kinds of magnetic oscillations, e.g. de Haas-van Alphen or Shubnikovde Haas oscillations (see [2]), as well as the so called Quantum Hall Effect or Fractional Hall Effect (see [1]). We will argue in the remaining parts of this article, that oscillatory phenomena are naturally built in the system (1), (5). It should also be mentioned that this system of equations, just as any other gauge theoretic system of equations, contains information about the topological quantum numbers. These could be later applied to modeling the QHE.

6. We want to understand basic features of solutions of the system (4), (5) in the case of two dimensional noncompact manifolds. In this case $\delta(f F)=0$ implies $\star F=\frac{B}{f}$ for a constant $B$. Also, in this case $d F=0$ 
and $F$ is a curvature of a certain connection $A$. Thus our system of equations reduces to

$$
-\triangle f+\frac{B^{2}}{f}=\nu f .
$$

Let us emphasize the fact that $\triangle f$ depends on the choice of geometry, more strictly - the Riemannian metric tensor $\left(g_{i j}\right)$.

We now show results of computer simulations for different choices of geometry. All figures present solutions $f$ of the equation (6) which are assumed to be radially symmetric, or to depend on one spatial variable only, and satisfy $f(0)=1, f^{\prime}(0)=0$. The parameters $B$ and $\nu$ are set to convenient values, but do not affect the qualitative properties of the pictures too radically. We point out that the shape of the derivative of $f$ in all cases resembles the distorted sinusoidal pattern. This pattern is characteristic for magnetic interactions of external fields with ferromagnetics. One of the figures shows how the distortion depends on $\nu$.

The last figures show dependence of radially symmetric solutions with $f(0)=1, f^{\prime}(0)=0$ on $\nu^{\frac{1}{2}}$, i.e. we plot $\mathrm{f}(0.5)$ against $\nu^{\frac{1}{2}}$ (fixed $B=1$, relative scale, undersampled data).

Let us also mention here that simulated solutions of the system (11), (5) on manifolds of three and four dimensions (with special Ansatzen like e.g. the t'Hooft Ansatz) display similar qualitative properties. 

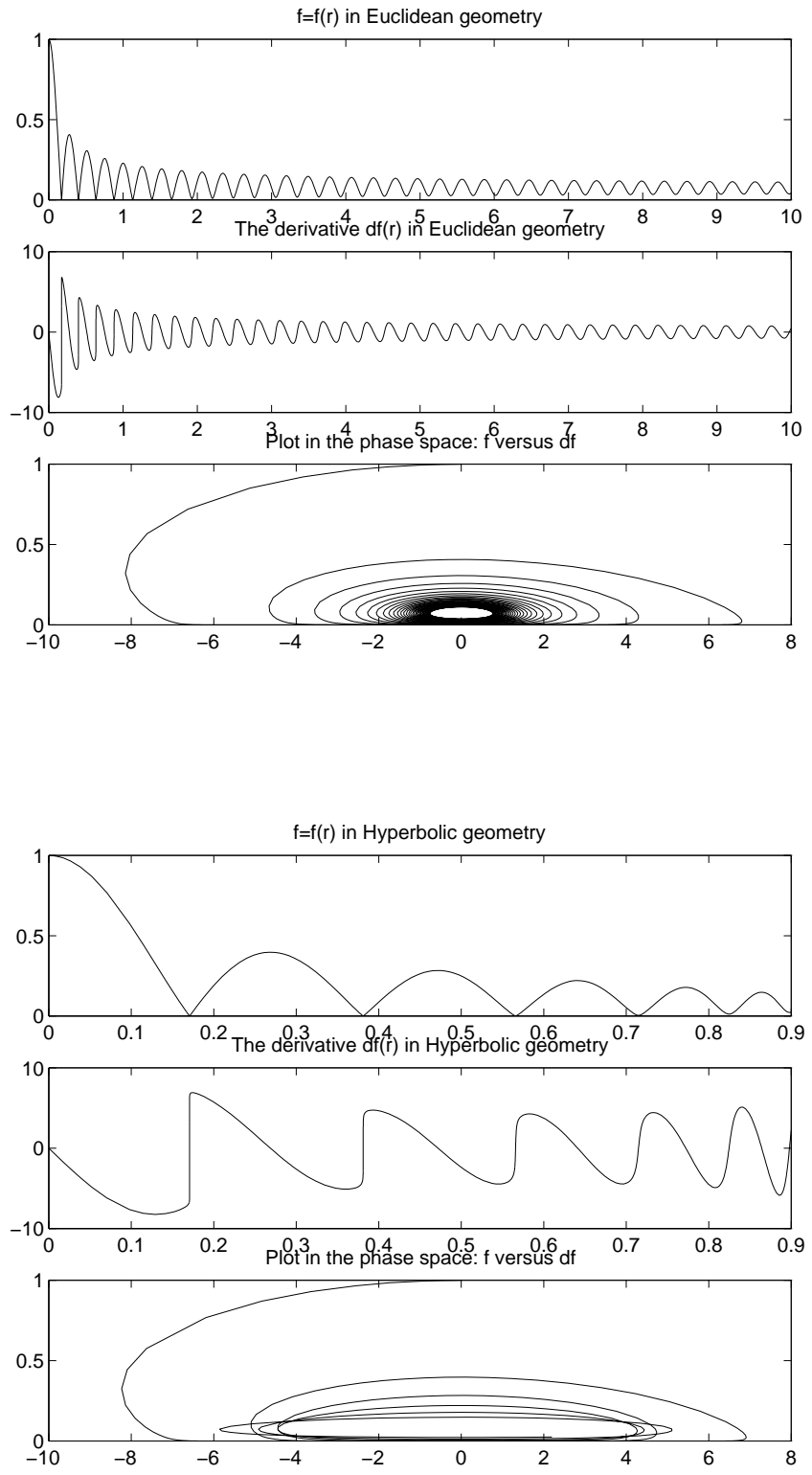

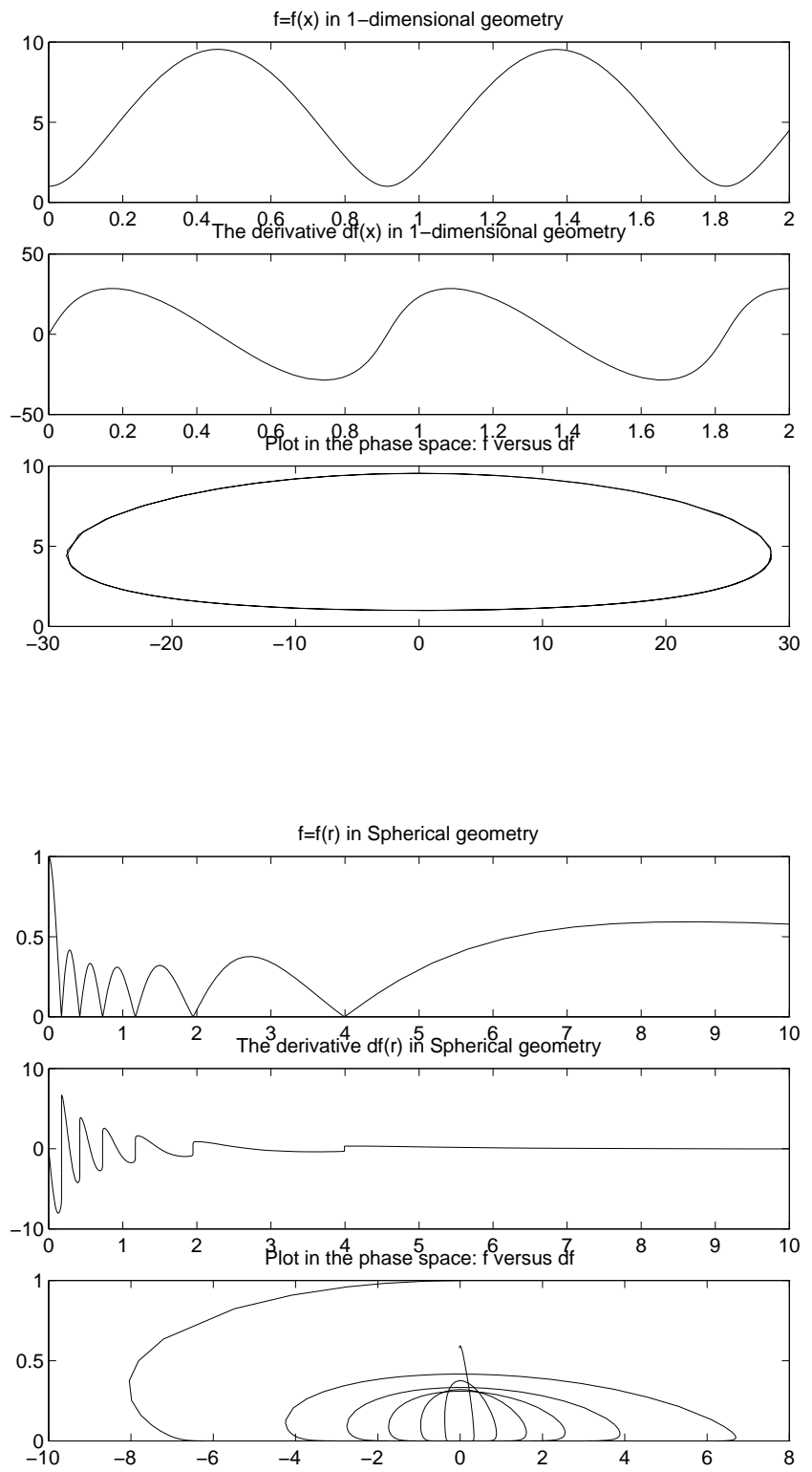

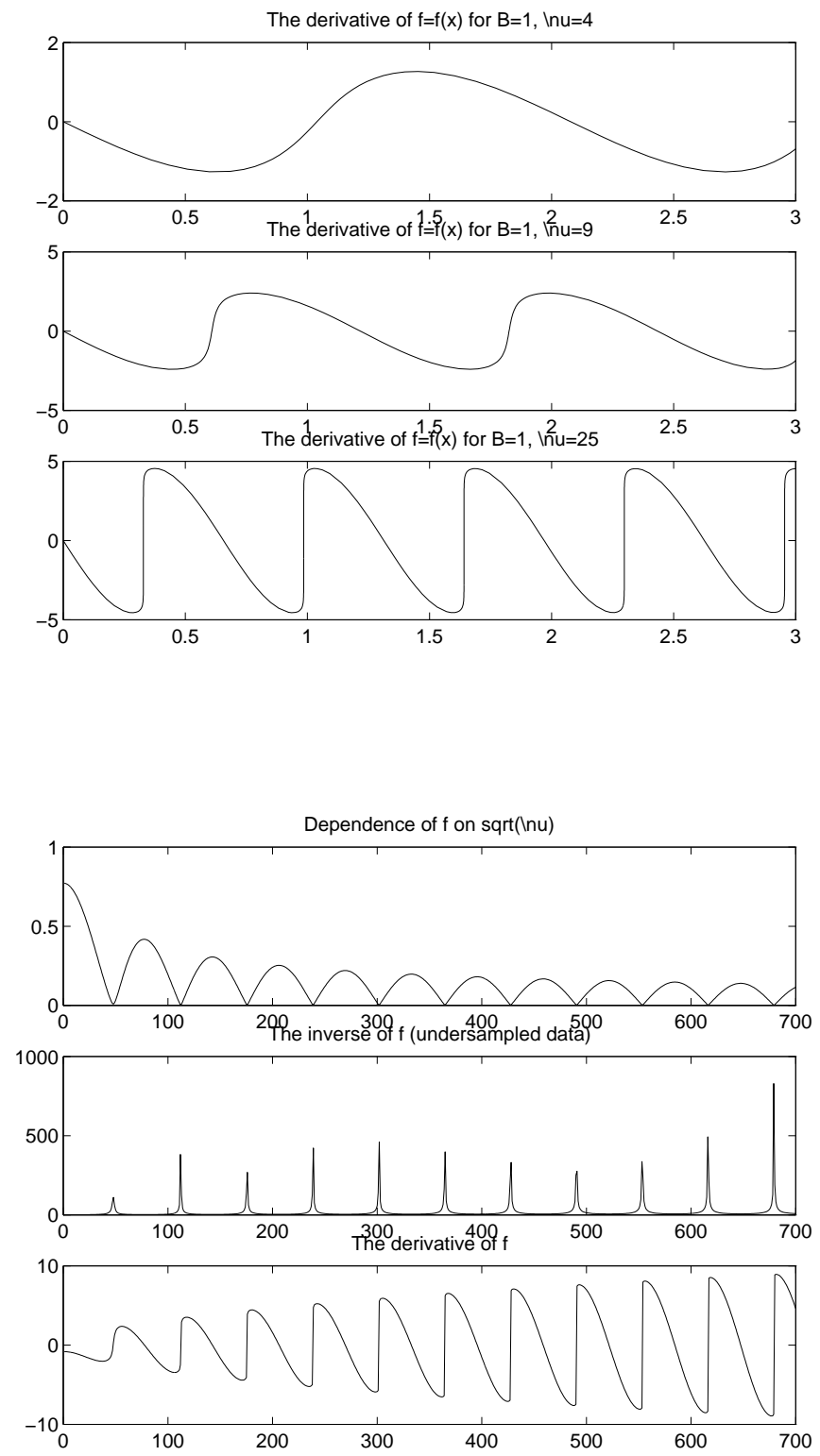

7. In conclusion, we want to shed some light on simple features of solutions of the system, considering it on a compact two-manifold $M$. In what follows, we assume for simplicity that $\operatorname{Vol}(M)=1$. Now, let $f$ be a solution of the equation (6). Since $F=\star \frac{B}{f}$ is the curvature of a 
connection on a principal $U(1)$-bundle, we know that the first Chern class (the topological quantum number) $\int F=N \in 2 \pi Z$, and therefore

$$
\int \frac{B}{f}=\int F=N \in 2 \pi Z \text {. }
$$

Let us also note that if $f$ satisfies the equation (5) and the condition (7), then so does each of its multiples (with a different value of $B$ ). Thus, we may assume without loss of generality that $\int \frac{1}{f}=1$, and the original equation becomes

$$
-\triangle f+\frac{N^{2}}{f}=\nu f
$$

Integrating both sides of the equation, we obtain $N^{2} \int \frac{1}{f}=\nu \int f$, and since $\int \frac{1}{f}=1, \operatorname{Vol}(M)=1$, we have by convexity of the function $x \longrightarrow$ $\frac{1}{x}$ that $\frac{N^{2}}{\nu}=\int f \geq \frac{1}{\int \frac{1}{f}}=1$, and so

$$
\nu \leq N^{2}
$$

Multiplying (6) by $f$ and integrating both sides again, we obtain

$$
\int|d f|^{2}+N^{2}=\nu \int f^{2} .
$$

On the other hand $\int f^{2} \geq\left(\int f\right)^{2}=\frac{N^{4}}{\nu^{2}}$, and combining this with the equation above, we finally obtain

Main Inequality 1 For solutions of (16) with the topological quantum number $N$, and such that $\int \frac{1}{f}=1$, we have

$$
\int|d f|^{2} \geq N^{2}\left(\frac{N^{2}}{\nu}-1\right) \geq 0,
$$

where both inequalities become identities if and only if $f$ is the constant solution.

Therefore one expects that the solutions for a fixed bundle become more "convolved" as the secondary quantum number $\nu$ gets smaller. It remains an open problem whether the set of eigenvalues $\nu$ is discrete on compact manifolds. 


\section{References}

[1] R. E. Prange, S. M. Girvin, Eds., The Quantum Hall Effect, SpringerVerlag, 1990

[2] D. Schoenberg, Magnetic Oscillations in Metals, Cambridge University Press, 1984

[3] A. Sowa, Abstracts of the AMS, Vol. 15, No. 3, p. 395, 1994

[4] A. Sowa, Geometric Unification of Yang-Mills and Shrödinger Equations, thesis, The City University of New York

[5] A. Sowa, On an Equation Arising From the Geometry of Riemannian Submersions, preprint 